\section{RMD Open}

Rheumatic \&

Musculoskeletal Diseases
To cite: Venerito V, Stefanizzi P, Fornaro $\mathrm{M}$, et al. Immunogenicity of BNT162b2 mRNA SARS$\mathrm{CoV}$-2 vaccine in patients with psoriatic arthritis on TNF inhibitors. RMD Open 2022;8:e001847. doi:10.1136/ rmdopen-2021-001847

- Additional supplemental material is published online only. To view, please visit the journal online (http://dx.doi.org/10. 1136/rmdopen-2021-001847).

W and PS are joint first authors

Received 27 July 2021

Accepted 5 December 2021

Check for updates

(C) Author(s) (or their employer(s)) 2022. Re-use permitted under CC BY-NC. No commercial re-use. See rights and permissions. Published by BMJ.

${ }^{1}$ Rheumatology Unit, Department of Emergency and Organ Transplantation, University of Bari Aldo Moro, Bari, Italy ${ }^{2}$ Hygiene and Preventive Medicine Unit, Department of Biomedical Science and Human Oncology, University of Bari Aldo Moro, Bari, Italy

Correspondence to Professor Giuseppe Lopalco; giuseppe.lopalco@uniba.it

\title{
Immunogenicity of BNT162b2 mRNA SARS-CoV-2 vaccine in patients with psoriatic arthritis on TNF inhibitors
}

\section{ABSTRACT}

Background Scanty data on the immunogenicity of the BNT162b2 vaccine in patients with psoriatic arthritis (PsA) on Tumor Necrosis Factor inhibitors (TNFi) have been published.

Objective To investigate the humoral response to BNT162b2 vaccination patients with PsA on TNFi, comparing immunogenicity with healthy controls. Methods Forty patients with classified PsA on TNFi undergoing vaccination with the BNT162b2 mRNA SARSCoV-2 vaccine (BioNTech/Pfizer) were enrolled. Fifteen days after the second shot, serum IgG levels against SARS-CoV-2 (Abbott ARCHITECT i2000SR, positivity cutoff $50 \mathrm{AU} / \mathrm{mL}$ ) were assayed in all patients. Clinimetrics and treatment data were gathered. TNFi treatment was not discontinued throughout the whole period, whereas methotrexate (MTX) was discontinued for 1 week after each shot in those on combination therapy. Sera from healthcare professionals were considered as healthy controls for 1:1 propensity score matching; any of them was taking medication.

Student's t-test and logistic regression were used for investigating differences in immunogenicity between groups and predictors of antibody response.

Results Clinical Disease Activity Index did not change before and after vaccination $(7.06 \pm 5.23$ to $7.10 \pm 5.27$, $\mathrm{p}=0.92$ ).

Patients with PsA achieved a positive anti-SARS-CoV-2 IgG level with a mean $( \pm S D)$ of $13794.44 \pm 15815.42 \mathrm{AU} /$ $\mathrm{mL}$. Although lower, the antibody level was not significantly different from matched controls (19227.4 \pm 11.8460 .45$ $\mathrm{AU} / \mathrm{mL}, \mathrm{p}=0.08$ ). In the overall sample, those on MTX $(12 / 80,15 \%)$ had a trend toward lower immune response $(p=0.07)$; glucocorticoid therapy $(11 / 80,13.8 \%)$ predicted lower antibody levels $(p=0.04)$.

Conclusions Continuing TNFi in patients with PsA throughout the vaccination did not hamper immunogenicity.

\section{INTRODUCTION}

Psoriatic arthritis (PsA) is an immunemediated inflammatory disease typically involving peripheral joints, enthesis, spine and skin. ${ }^{1}$ Several studies reported an

\section{Key messages}

Vaccination against COVID-19 should be strongly considered for most patients with psoriatic arthritis (PsA). Growing evidence suggests that diseasemodifying antirheumatic drugs should be continued before and after vaccination.

- This study confirms that TNF inhibitors (TNFi) in patients with PsA during vaccination with BNT162b2 are safe and are not associated with hampered immune response.

- TNFi may not be discontinued in patients with PSA during vaccination with BNT162b2.

increased risk of infection in patients with rheumatic diseases, mainly due to untreated disease activity, comorbidities and/or longterm use of immunosuppressants. ${ }^{2}$ Patients with inflammatory diseases, including psoriasis and PsA, when analysed as a combined group, might have a slightly increased risk of death from COVID-19 compared with healthy people. ${ }^{3}$ Continuing treatment may help to prevent hospitalisation due to COVID19, possibly by keeping disease activity in check. $^{45}$ Vaccination against COVID-19 is a valid strategy for most patients with rheumatic diseases, including PsA. ${ }^{56}$ Nevertheless, the immune response to vaccination can be somewhat reduced in patients with several disease-modifying antirheumatic drugs (DMARDs). ${ }^{5}$ Of note, patients on immunosuppressive medications were excluded from phase III clinical trials of mRNA vaccines, ${ }^{7}$ so it is unclear whether DMARDs should be continued before and after vaccination. Despite several real-world studies showing successful immunogenicity of mRNA vaccine in immunocompetent people, a recent report suggested that either TNF inhibitors (TNFi) or methotrexate (MTX) might hamper immune response to anti-SARS-CoV-2 in patients with 
inflammatory diseases. ${ }^{6} 8$ To date, scanty data on the immunogenicity of the BNT162b2 mRNA vaccine in patients with PsA on TNFi have been published. Notably, recent reports are characterised by within-cohort heterogeneity about the timing of the anti-SARS-CoV-2 IgG test after the second shot. ${ }^{69}$ Furthermore, the wide variability of the protocols of discontinuation of csDMARDs and steroid therapy at vaccination also is noticeable.

Our study aimed at investigating the humoral immune response to BNT162b2 vaccination in a cohort of patients with PsA on TNFi with homogeneous disease characteristics and treatment comparing vaccine immunogenicity with healthy controls.

\section{METHODS}

Patients with classified PsA according to ClASsification criteria for Psoriatic ARthritis (CASPAR) ${ }^{10}$ on TNFi followed at a PsA clinic of our tertiary centre were longitudinally observed from April to June 2021. Our institution set up a vaccination campaign during which patients with PsA received two shots of the BNT162b2 mRNA SARS-CoV-2 vaccine (BioNTech/Pfizer) on 24 April and 15 May. Our study included all consecutive patients who underwent vaccination during such a campaign. Fifteen days after the second shot, all patients with PsA underwent IgG test against the S1 domain of the spike protein of SARS-CoV-2 (Abbott ARCHITECT i2000SR, positivity cut-off $50 \mathrm{AU} / \mathrm{mL}$ ); blood samples were collected and analysed at the same institution's laboratory. TNFi treatment and non-MTX csDMARDs were not discontinued throughout the whole period. In all patients, MTX was held the week after each shot, in observance of the American College of Rheumatology Guidance to the timing of vaccination, adopted in our hospital. ${ }^{11}$ Patients were contacted to record when they took the last MTX dose before vaccination.

Demographic and clinical characteristics, including disease phenotypes, Disease Activity in PsA (DAPSA), Psoriasis Area Severity Index (PASI), Clinical Disease Activity Index (CDAI), Morisky Medication Adherence Score on 4 Items (MMAS-4) and PsA Impact of Disease (PsAID12), ${ }^{12}$ were recorded at baseline consultation before vaccination. At the same time, patients were asked for prior COVID-19 and/or related symptoms.

The assessment of CDAI was also repeated at the time of anti-SARS-CoV-2 IgG testing. We also recorded any vaccine reaction and/or adverse event during the observation period.

Sera from 59 healthcare professionals employed at our institution, gathered 15 days after the second BioNTech/Pfizer vaccine shot from January 2021 to February 2021, were considered as healthy controls for $1: 1$ nearestneighbour Propensity Score (PS) matching. ${ }^{13}$ PS is an epidemiological tool used for the adjustment of nonrandomised longitudinal studies. It is a conditional probability of being exposed to a disease given a set of covariates. In brief, this was carried out using the patients' age and gender, with a selected calliper of 0.2. A proper assessment of postmatching balance was also carried out as previously described. ${ }^{13}$ As a result of PS matching, 19 controls were lost, leading to a postmatched sample of 80 individuals, consisting of 40 patients in the PsA group and 40 patients in the control group.

We assessed the difference in anti-SARS-CoV-2 IgG levels between groups with Student's t-test. Paired t-test and McNemar's test were used to determine the difference between mean CDAI Score and remission rate at different time points, respectively. Associations of recorded covariates with IgG levels and CDAI scores after vaccination were investigated using linear regression.

\section{RESULTS}

Mean age $( \pm$ SD) of patients with PsA was $52.85 \pm 10.41$ years; $22 / 40(55 \%)$ were female, with a mean disease duration of $11 \pm 9.0$ years. Oligoarticular involvement was the prevalent phenotype $(22 / 40,55 \%)$, with a minority of patients being affected with axial disease $(6 / 40,15 \%)$. By first shot, 12/40 patients with PsA (30\%) were on MTX combotherapy at a mean dose of $14.79 \pm 3.27 \mathrm{mg}$ weekly. Patients took the last MTX dose a median of 3 days (min-max 1-6) before vaccination. Overall, 11 out of $40(27.5 \%)$ were on glucocorticoids at a mean dose of $4.66 \pm 1.67$ prednisone equivalent. Mean DAPsA was 11.34 \pm 7.81. Patients with PsA in our cohort had mild skin involvement with a mean PASI of $0.56 \pm 1.01$ and moderate life impact (mean PsAID12 3.94 \pm 2.40 ). Patients with PsA declared high medication adherence (median MMAS-4 0 , min-max 0-1). None of them reported prior COVID-19 or SARS-CoV-2 infection-related symptoms.

Mean age of PS-matched controls was $50.80 \pm 12.16$ years $(\mathrm{p}=0.42)$ with similar gender distribution (female $18 / 4045 \%, \mathrm{p}=0.37$ ). Patient characteristics are detailed in table 1 . None of the controls was taking immunosuppressants or glucocorticoids at vaccination. At the first shot, fever was the most frequent symptom accompanying vaccination $(7 / 40,17.50 \%)$, followed by arthralgia and lymphadenopathy $(2 / 40,5 \%$, for both). At the second shot, the percentage of patients experiencing joint pain was significantly higher $(9 / 40,22.50 \%, \mathrm{p}=0.007)$, with the fever still occurring in the $17.50 \%(7 / 40)$ of the cohort (table 2).

No changes of clinical disease activity between baseline (CDAI 7.06 \pm 5.23 ) and after the complete vaccination (CDAI 7.10 $\pm 5.27, \mathrm{p}=0.92$ ) were detected. Consistently, the CDAI-based remission rate remained similar (28/40 vs $23 / 40, p=0.31$ ). All patients with PsA showed a positive immune response with a mean anti-SARS-CoV-2 IgG level of $13794.44 \pm 15815.42 \mathrm{AU} / \mathrm{mL}$. Although quantitatively lower, this was not significantly different from matched controls' titre, which was $19227.4 \pm 11.8460 .45 \mathrm{AU} / \mathrm{mL}$ $(\mathrm{p}=0.08$, figure 1$)$.

No difference was shown between patients with PsA with or without MTX $(9331.80 \pm 12131.09$ vs $15707 \pm 16991.04 \mathrm{AU} / \mathrm{mL}$ respectively, $\mathrm{p}=0.24 ; 95 \% \mathrm{CI}$ 
Table 1 Patient characteristics

\begin{tabular}{|c|c|c|c|c|}
\hline \multirow{3}{*}{ Age, mean (SD) } & \multicolumn{2}{|c|}{ Last visit before first shot } & \multicolumn{2}{|c|}{15 days after the second shot } \\
\hline & Av.Obs. & & Av.Obs & \\
\hline & 40 & $52.85(10.41)$ & & \\
\hline Female, n (\%) & 40 & $22(55)$ & & \\
\hline Disease duration, years, mean (SD) & 40 & $11(9.01)$ & & \\
\hline Axial, $\mathrm{n}(\%)$ & 40 & $6(15)$ & & \\
\hline Psoriasis, $\mathrm{n}(\%)$ & 40 & $33(82.5)$ & & \\
\hline Polyarticular, n (\%) & 40 & $18(45)$ & & \\
\hline PsAID12, mean (SD) & 40 & $3.94(2.4)$ & & \\
\hline MMAS-4, median (min-max) & 38 & $0(0-1)$ & & \\
\hline DAPsA at vaccine, mean (SD) & 40 & $11.34(7.81)$ & & \\
\hline PASI, mean (SD) & 40 & $0.56(1.01)$ & & \\
\hline CDAI, mean (SD) & 40 & $7.06(5.23)$ & 40 & $7.10^{*}(5.27)$ \\
\hline CDAI remission/LDA, n (\%) & 40 & $28(73)$ & 40 & $23 \dagger(67.5)$ \\
\hline Glucocorticoid, n (\%) & 40 & $11(27.5)$ & & \\
\hline Glucocorticoid dose, mean (SD) & 11 & $4.66(1.97)$ & & \\
\hline Combotherapy, n (\%) & 40 & $19(42.5)$ & & \\
\hline Methotrexate, n (\%) & 40 & $12(40)$ & & \\
\hline Sulfasalazine, $\mathrm{n}(\%)$ & 40 & $7(17.5)$ & & \\
\hline bDMARD treatment first line, $\mathrm{n}(\%)$ & 40 & $7(17.5)$ & & \\
\hline bDMARD treatment second line, $\mathrm{n}(\%)$ & 40 & $30(75)$ & & \\
\hline bDMARD treatment third line, $\mathrm{n}(\%)$ & 40 & $3(7.5)$ & & \\
\hline Adalimumab, n (\%) & 40 & $13(32.5)$ & & \\
\hline Infliximab, n (\%) & 40 & $11(27.5)$ & & \\
\hline Certolizumab, n (\%) & 40 & $7(17.5)$ & & \\
\hline Etanercept, n (\%) & 40 & $7(17.5)$ & & \\
\hline Golimumab, n (\%) & 40 & $2(5)$ & & \\
\hline
\end{tabular}

*Paired t-test, $\mathrm{p}=0.92$.

†McNemar's test, $p=0.31$.

Av.Obs., available observations; bDMARD, biologic disease-modifying antirheumatic drugs; CDAl, Clinical Disease Activity Index; DAPsA, Disease Activity in Psoriatic Arthritis; LDA, low disease activity; MMAS-4, Morisky Medication Adherence Score on 4 Items; PASI, Psoriasis Area Severity Index; PsAID12, Psoriatic Arthritis Impact of Disease.

-17368.82 to 4618.42$)$, also after adjusting for sulfasalazine treatment $(\mathrm{p}=0.51 ; 95 \% \mathrm{CI}-20997.61$ to 10682.96). The same findings were also observed when patients with PsA taking glucocorticoids were compared with those without $(9974.282 \pm 12357.45$ vs $15243.47 \pm 16909.93 \mathrm{AU} / \mathrm{mL}, \mathrm{p}=0.35 ; 95 \% \mathrm{CI}-16$ 623.61 to 6085.24$)$. Considering the whole PS-matched sample, hence including healthy controls, MTX use was not associated with a lower anti-SARS-CoV-2 IgG titre $(\mathrm{p}=0.07 ; 95 \%$ CI -17116.44 to 224.69$)$; the same was true for sulfasalazine ( $\mathrm{p}=0.67 ; 95 \%$ CI -8738.55 to $5652.92)$. In contrast, glucocorticoid use was a predictor of lower immunogenicity ( $\mathrm{p}=0.04 ; 95 \%$ CI -8800.32 to $-118.76)$. We also investigated whether disease activity flared because of MTX discontinuation after vaccination shots. In this regard, MTX discontinuation did not

Table 2 Vaccine reactions

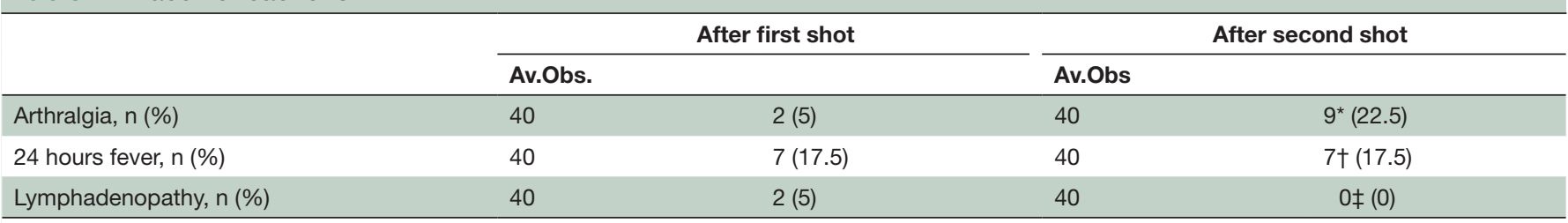

*McNemar's test, $\mathrm{p}=0.007$.

$\dagger \chi^{2}$ test, $p=1$.

$\neq \chi^{2}$ test, $\mathrm{p}=0.151$

Av.Obs., available observations. 


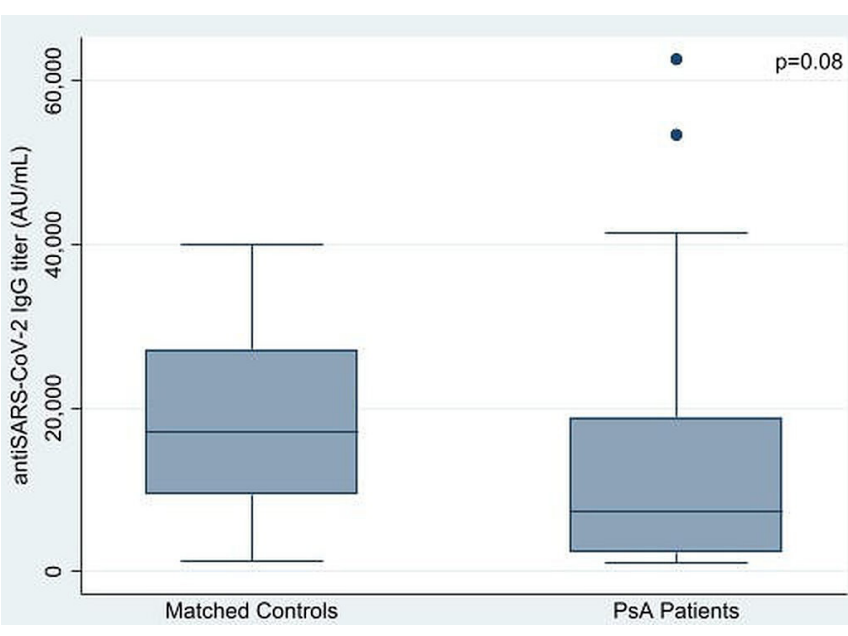

Figure 1 Anti-SARS-CoV-2 IgG titers of patients with Psoriatic Arthritis (PsA) and matched healthy controls.

predict a higher CDAI Score $(\mathrm{p}=0.09 ; 95 \%$ CI -2.16 to $5.41)$.

\section{DISCUSSION}

This study analyses the immunogenicity of the BNT162b2 vaccine in patients with PsA on TNFi, comparing them to PS-matched controls. An arbitrary IgG serum level representative of vaccine efficacy that was not defined as robust evidence has not been provided yet. To clearly depict the effect of TNFi on vaccine immune response, only MTX was discontinued for 1 week after each shot. Like recent reports, ${ }^{9}$ the immunogenicity of $\mathrm{BNT} 162 \mathrm{~b} 2$ was not hindered in patients with PsA on TNFi compared with healthy controls. In a recent report from Mahil et al on patients with psoriasis, the rate of serological conversion of those on MTX was quite low, contrasting with our results and with a previous study in which patients who were taking MTX showed adequate humoral responses after the first shot of BNT162b2. ${ }^{9} 14$

Unlike our cohort, adherence to MTX was confirmed in all patients in the study by Mahil et $a l,{ }^{9}$ without any pause in the vaccination period. On this basis, we can argue that MTX not leading to decreased IgG titers might be a consequence of its discontinuation 1 week after each shot. We also showed that neither vaccination nor MTX discontinuation impacted PsA disease activity, which remained stable. Consistently with recent literature, ${ }^{15}$ glucocorticoid use at vaccine administration might lead to lower IgG titers.

Among study limitations, we acknowledge the small sample size. Additionally, we cannot exclude the fact that a few patients got asymptomatic SARS-CoV-2 infection at any time before vaccination, as immunisation status was not assessed. Furthermore, we did not perform a postvaccination serum viral neutralising test.

Notably, our analysis was neither intended nor powered to show the difference between patients assuming or not assuming background MTX mainly for the lack of comparator group continuing treatment.

Our study has some strengths. First, PS matching on prospectively gathered data, together with homogeneous treatment management, allowed to mitigate bias due to non-randomisation. Moreover, the timing of sera sampling was the same for all patients, and to reduce the within-cohort variability, an anti-SARSCoV-2 IgG assay was run once for all the samples at the same laboratory.

In conclusion, continuing TNFi therapy in patients with PsA throughout the vaccination period was not associated with hampered immune response and it was safe. Although MTX was not associated with decreased IgG titers, more data are needed to clarify whether holding MTX after vaccination may lead to optimal immunogenicity.

\section{Twitter Vincenzo Venerito @vincevenerito}

Contributors Study design: W, GL, PS and ST. Data gathering and manuscript editing: W, GL and PS. Data analysis: VV, PS and ST. Supervision: FI and ST.

Funding The authors have not declared a specific grant for this research from any funding agency in the public, commercial or not-for-profit sectors.

Competing interests None declared.

Patient consent for publication Not applicable.

Ethics approval This study follows the Declaration of Helsinki and received local ethics committee approval (Comitato Etico Interregionale Policlinico di Bari) as part of BIOPURE Study (Approval n.5940). Participants gave informed consent to participate in the study before taking part.

Provenance and peer review Not commissioned; externally peer reviewed.

Data availability statement Complete dataset is available upon request and provided local Ethics Committee approval for sharing.

Supplemental material This content has been supplied by the author(s). It has not been vetted by BMJ Publishing Group Limited (BMJ) and may not have been peer-reviewed. Any opinions or recommendations discussed are solely those of the author(s) and are not endorsed by BMJ. BMJ disclaims all liability and responsibility arising from any reliance placed on the content. Where the content includes any translated material, BMJ does not warrant the accuracy and reliability of the translations (including but not limited to local regulations, clinical guidelines, terminology, drug names and drug dosages), and is not responsible for any error and/or omissions arising from translation and adaptation or otherwise.

Open access This is an open access article distributed in accordance with the Creative Commons Attribution Non Commercial (CC BY-NC 4.0) license, which permits others to distribute, remix, adapt, build upon this work non-commercially, and license their derivative works on different terms, provided the original work is properly cited, appropriate credit is given, any changes made indicated, and the use is non-commercial. See: http://creativecommons.org/licenses/by-nc/4.0/

\section{ORCID iDs}

Vincenzo Venerito http://orcid.org/0000-0002-2573-5930

Fabio Cacciapaglia http://orcid.org/0000-0001-7479-4462

Simone Perniola http://orcid.org/0000-0001-6918-4731

\section{REFERENCES}

1 lannone $\mathrm{F}$, Nivuori M, Fornaro M, et al. Comorbid fibromyalgia impairs the effectiveness of biologic drugs in patients with psoriatic arthritis. Rheumatology 2020;59:1599-606.

2 Furer V, Rondaan C, Heijstek MW, et al. 2019 update of EULAR recommendations for vaccination in adult patients with autoimmune inflammatory rheumatic diseases. Ann Rheum Dis 2020;79:39-52.

3 Hyrich KL, Machado PM. Rheumatic disease and COVID-19: epidemiology and outcomes. Nat Rev Rheumatol 2021;17:71-2.

4 Gianfrancesco M, Hyrich KL, Al-Adely S, et al. Characteristics associated with hospitalisation for COVID-19 in people 
with rheumatic disease: data from the COVID-19 global rheumatology alliance physician-reported registry. Ann Rheum Dis 2020;79:859-66.

5 Fagni F, Simon D, Tascilar K, et al. COVID-19 and immunemediated inflammatory diseases: effect of disease and treatment on COVID-19 outcomes and vaccine responses. Lancet Rheumatol 2021;3:e724-36.

6 Haberman RH, Herati R, Simon D, et al. Methotrexate hampers immunogenicity to BNT162b2 mRNA COVID-19 vaccine in immunemediated inflammatory disease. Ann Rheum Dis 2021;80:1339-44.

7 Polack FP, Thomas SJ, Kitchin N, et al. Safety and efficacy of the BNT162b2 mRNA Covid-19 vaccine. N Engl J Med 2020;383:2603-15.

8 Kennedy NA, Lin S, Goodhand JR, et al. Infliximab is associated with attenuated immunogenicity to BNT162b2 and ChAdOx1 nCoV-19 SARS-CoV-2 vaccines in patients with IBD. Gut 2021;70:1884-93.

9 Mahil SK, Bechman K, Raharja A, et al. The effect of methotrexate and targeted immunosuppression on humoral and cellular immune responses to the COVID-19 vaccine BNT162b2: a cohort study. Lancet Rheumatol 2021;3:e627-37.

10 Taylor W, Gladman D, Helliwell P, et al. Classification criteria for psoriatic arthritis: development of new criteria from a large international study. Arthritis Rheum 2006;54:10.1002/ art.21972:2665-73.

11 Curtis JR, Johnson SR, Anthony DD, et al. American College of rheumatology guidance for COVID-19 vaccination in patients with rheumatic and musculoskeletal diseases: version 1. Arthritis Rheumatol 2021;73:1093-107.

12 Gladman DD, Mease PJ, Krueger G, et al. Outcome measures in psoriatic arthritis. J Rheumatol 2005;32:2262-9.

13 Park JW, Curtis JR, Moon J, et al. Prophylactic effect of trimethoprim-sulfamethoxazole for Pneumocystis pneumonia in patients with rheumatic diseases exposed to prolonged high-dose glucocorticoids. Ann Rheum Dis 2018;77:10.1136/annrheumdis2017-211796:644-9.

14 Boyarsky BJ, Ruddy JA, Connolly CM, et al. Antibody response to a single dose of SARS-CoV-2 mRNA vaccine in patients with rheumatic and musculoskeletal diseases. Ann Rheum Dis 2021:1098-9.

15 Bugatti S, De Stefano L, Balduzzi S. Methotrexate and glucocorticoids, but not anticytokine therapy, impair the immunogenicity of a single dose of the BNT162b2 mRNA COVID-19 vaccine in patients with chronic inflammatory arthritis. Ann Rheum Dis 2021:annrheumdis-2021-220862. 